\title{
Crystalline Sponges as a Sensitive and Fast Method for Metabolite Identification: Application to Gemfibrozil and its Phase I and II Metabolites $^{\mathbb{S}}$
}

\author{
Lara Rosenberger, Carolina von Essen, Anupam Khutia, Clemens Kühn, Klaus Urbahns, \\ Katrin Georgi, Rolf W. Hartmann, and Lassina Badolo

\begin{abstract}
Discovery and Development Technologies (DDTech) (L.R., K.U., K.G., L.B.) and Innovation Center (C.v.E., A.K., C.K.), Merck KGaA, Darmstadt, Germany; and Department of Drug Design and Optimization (DDOP), Helmholtz-Institute for Pharmaceutical Research Saarland (HIPS)-Helmholtz Centre for Infection Research (HZI), Saarbrücken, Germany (L.R., R.W.H.)
\end{abstract}

Received March 3, 2020; accepted April 20, 2020

\begin{abstract}
Understanding the metabolism of new drug candidates is important during drug discovery and development, as circulating metabolites may contribute to efficacy or cause safety issues. In the early phase of drug discovery, human in vitro systems are used to investigate human relevant metabolism. Though conventional techniques are limited in their ability to provide complete molecular structures of metabolites (liquid chromatography mass spectrometry) or require a larger amount of material not available from in vitro incubation (nuclear magnetic resonance), we here report for the first time the use of the crystalline sponge method to identify phase I and phase II metabolites generated from in vitro liver microsomes or $\mathbf{S 9}$ fractions. Gemfibrozil was used as a test compound. Metabolites generated from incubation with microsomes or $\mathrm{S} 9$ fractions, were fractionated using online fraction collection. After chromatographic purification and fractionation of the generated metabolites, single crystal X-ray diffraction of crystalline sponges was used to identify the structure
\end{abstract}

of gemfibrozil metabolites. This technique allowed for complete structure elucidation of $5^{\prime}-\mathrm{CH}_{2} \mathrm{OH}$ gemfibrozil (M1), 4'-OH gemfibrozil (M2), 5'-COOH gemfibrozil (M3), and the acyl glucuronide of gemfibrozil, 1-O- $\beta$-glucuronide (M4), the first acyl glucuronide available in the Cambridge Crystallographic Data Centre. Our study shows that when optimal soaking is possible, crystalline sponges technology is a sensitive (nanogram amount) and fast (few days) method that can be applied early in drug discovery to identify the structure of pure metabolites from in vitro incubations.

\section{SIGNIFICANCE STATEMENT}

Complete structure elucidation of human metabolites plays a critical role in early drug discovery. Low amounts of material (nanogram) are only available at this stage and insufficient for nuclear magnetic resonance analysis. The crystalline sponge method has the potential to close this gap, as demonstrated in this study.

\section{Introduction}

During the process of drug discovery and development, assessing the identity of drug metabolites is crucial to understand the contribution of metabolites to efficacy or safety (Nedderman, 2009; Zhang et al., 2009). Guidelines have been drafted by regulatory agencies (EMA, 2013; FDA, 2016) to help the pharmaceutical industry to apply the right strategy regarding metabolites in safety assessment. The key element in all these guidances is to protect patients and healthy human volunteers from potentially toxic metabolites with a focus on human metabolites that are either absent in preclinical species or circulate in these animals at much lower concentrations than in humans (Ma and Chowdhury, 2011). It is therefore of paramount importance during drug development to gain an understanding of metabolism of new chemical entities in humans as early as possible. To achieve this, in vitro systems from human origin (e.g., microsomes or hepatocytes) are used to metabolize the new active

https://doi.org/10.1124/dmd.120.091140.

S This article has supplemental material available at dmd.aspetjournals.org. pharmaceutical ingredients (API) in drug discovery research (Brandon et al., 2003; Fasinu et al., 2012). Samples generated from these systems are analyzed with liquid chromatography (LC)-mass spectrometry (MS) of different types [e.g., tandem MS (MS ${ }^{2}$ ) and tandem time-of-flight] by drawing inferences from fragmentation patterns to assess metabolism (Prakash et al., 2007; Prasad et al., 2011). Although MS techniques have reached extreme sensitivity, these techniques fail in many situations, e.g., to identify the complete structure of metabolites. This forces scientists to use NMR, a less sensitive technique, requiring larger amounts of material (usually microgram to milligram range) (Murai et al., 2004), which is therefore not always suitable for analyzing samples from in vitro origin.

Besides NMR and LC-MS, another technique would be single crystal $\mathrm{X}$-ray diffraction (XRD), a direct technique that provides structural information at the atomic level by measuring electron density as diffraction pattern of a single crystal (Massa, 2004). Single crystal XRD can even provide information on the absolute configuration of chiral centers but has the intrinsic limitation because of requirements of single crystals of suitable sizes and shape. The process of crystallization

ABBREVIATIONS: API, active pharmaceutical ingredient; CS, crystalline sponge; CS-XRD, crystalline sponge method; DME, 1,2-dimethoxyethane; HPLC, high performance liquid chromatography; LC, liquid chromatography; MS, mass spectrometry; MS $^{2}$, tandem mass spectrometry; $R_{\text {int }}$ internal $R$-value $R_{1}$ residual factor tpt, 2,4,6-tris(4-pyridyl)-1,3,5-triazine; UDPGA, uridine 5'-diphosphoglucuronic acid; UPLC, ultraperformance liquid chromatography; $\mathrm{XRD}$, X-ray diffraction. 
is a time-consuming procedure that requires availability of sufficient amounts of materials and cannot be performed with amorphous solids, liquids, or volatile analytes. One approach to overcome the limitation of crystallization is the application of microcrystal electron diffraction from powder (Jones et al., 2018; Kunde and Schmidt, 2019). This technique requires solid material at a higher amount.

For structural elucidation of metabolites in solution, a method with the information density of X-ray crystallography and the sensitivity in the nanogram range is needed for human-related metabolites at this early stage of drug development research. In 2013, Makoto Fujita published a new technique commonly known as "crystalline sponge method" (CSXRD), which enables crystal structure determination without crystallization with only a nanogram to few microgram amount of analytes (Inokuma et al., 2013). In one specific case, only $80 \mathrm{ng}$ of material were sufficient to clearly elucidate the X-ray structure of the analyte, and presumably even smaller amounts are possible for synchrotron X-ray diffraction instruments. The CS-XRD uses pre-existing crystals of porous metal coordination complexes $\left[\left(\mathrm{ZnX}_{2}\right)_{3} \cdot(\mathrm{tpt})_{2} \cdot \mathrm{x}(\text { solvent })\right]_{\mathrm{n}}$ ( $\mathrm{X}=\mathrm{Cl}$; tpt=2,4,6-tris(4-pyridyl)-1,3,5-triazine), which can absorb organic molecules in its pores and make them observable by conventional single crystal X-ray crystallography. Via diffusion ("soaking"), the analyte ("guest") is absorbed into the pores of porous CS and regularly ordered by intermolecular, noncovalent interactions ("guest soaking") (Inokuma et al., 2013; Inokuma et al., 2016; Sakurai et al., 2017). As a result, the repetitive positioning of the analytes in each pore of the framework serves for structural analysis via X-ray diffraction. For a successful CS-XRD experiment, regular order of the analyte is important. Functionalization of the APIs (e.g., hydroxylation, demethylation) increases the type of noncovalent interactions with the framework and therefore increases the chances of regular order. This even works for otherwise noncrystallizable compounds (Hoshino et al., 2016). The CSXRD has been shown to be able to successfully elucidate the structure of APIs (Sakurai et al., 2017), products from biosynthesis catalyzed by cytochrome P450 enzymes (Morita et al., 2020), natural plant products (Wada et al., 2018), and metabolic products prepared by reductive dichlorination of an insecticide as well as keton reduction of a steroid hormone with baker's yeast (Inokuma et al., 2016), but it has not been applied for drug-related material generated by incubation of API's with human or animal tissues before.

In this study, we apply the crystalline sponge technology for complete structure identification of gemfibrozil phase I and phase II metabolites after incubation with liver microsomes or S9. Rat systems were used, however, and the formation of the same metabolites were confirmed in human liver S9.

\section{Materials and Methods}

Chemicals and Reagents. Gemfibrozil (Fig. 1) was purchased from Acros Organics (NJ), and its metabolite 4'OH gemfibrozil was synthesized from WuXi AppTec Co., Ltd. (Wuhan, China). Gemfibrozil glucuronide was purchased from Toronto Research Chemicals (Toronto, Canada). Di-potassium hydrogen phosphate, potassium dihydrogen phosphate, magnesium chloride hexahydrate, dimethylsulfoxide, cyclohexane, methanol, nitrobenzene, nicotinamide adenine dinucleotide phosphate disodium salt (NADP- $\mathrm{Na}_{2}$ ), water (ultra-high-performance LC-MS grade), and acetonitrile (ultra-high-performance LC-MS grade) were purchased from Merck KGaA (Darmstadt, Germany). Zinc chloride, 1,2dimethoxyethane (DME), alamethicin, uridine 5'-diphosphoglucuronic acid trisodium salt (UDPGA- $\mathrm{Na}_{3}$ ), and formic acid were purchased from Sigma Aldrich Chemie GmbH (Steinheim, Germany). Dihydronicotinamide adenine dinucleotide phosphate tetrasodium salt $\left(\mathrm{NADPH}-\mathrm{Na}_{4}\right)$ was purchased from AppliChem (Darmstadt, Germany), and 2,4,6-tri(4-pyridyl)-1,3,5-triazine (tpt) was purchased from abcr GmbH (Karlsruhe, Germany). Female WistarHan rat liver microsomes (R6500, pool of 225), female rat liver S9 (R3500.S9, pool of 100), and mixed gender human liver S9 (H0620.S9, pool of 50) were obtained from Sekisui XenoTech (Kansas City), and mixed gender human liver microsomes (Ultrapool, pool of 150) were purchased from Corning (Corning)

Metabolism of Gemfibrozil by Rat/Human Liver Microsomes, Rat/ Human Liver S9. The oxidation reaction of gemfibrozil was conducted with rat liver microsomes $(0.5 \mathrm{mg} / \mathrm{ml})$ in $50 \mathrm{mM}$ potassium phosphate buffer $(\mathrm{pH} 7.4)$ containing $1 \mathrm{mM}$ magnesiumchloride and $20 / 200 \mu \mathrm{M}$ substrate. After 5 minutes of preincubation $\left(37^{\circ} \mathrm{C}, 150 \mathrm{rpm}\right)$, the reaction was initiated by the addition of NADPH $(1.5 \mathrm{mM})$ dissolved in $50 \mathrm{mM}$ potassium buffer, and the mixture was incubated for another 3 hours (final volume: $5.40 \mathrm{ml}$ ). The reaction was terminated by adding one volume of cold acetonitrile and then centrifuged ( $4000 \mathrm{~g}, 1$ hour, $\left.4^{\circ} \mathrm{C}\right)$ to sediment the precipitated proteins. The aliquots $(100 \mu \mathrm{l})$ of the supernatant were injected onto high-performance LC (HPLC)-MS for separation and fractionation.

The oxidation of gemfibrozil with rat and human liver S9 fraction was conducted similar to the incubation with microsomes. Differences were the protein concentration of $1.0 \mathrm{mg} / \mathrm{ml}$, the addition of NADPH and $\mathrm{NADP}^{+}(1.5$ $\mathrm{mM})$ as cofactors, and the incubation time (6 hours).

To generate gemfibrozil glucuronide, human liver microsomes were treated with alamethicin $\left(4^{\circ} \mathrm{C}, 20\right.$ minutes, final concentration: $\left.25 \mu \mathrm{g} / \mathrm{ml}\right)$, a channelforming peptide to increase the metabolite formation. The reaction was initiated by the addition of UDPGA $(1.5 \mathrm{mM})$ as cofactor.

HPLC-MS. The supernatants were analyzed on an Acquity Arc HPLC system combined with an isocratic solvent manager, a single quadrupole MS, and a fraction manager (Waters Corporation, Milford). The LC system included a quaternary solvent manager, a sample manager, and a 2998 photodiode array detector. The isocratic solvent manager was used with a 1:50 splitter. Samples were analyzed with electrospray ionization mass spectrometry in the negative ion mode. The following source parameters were used: probe temperature, $600^{\circ} \mathrm{C}$; capillary voltage, $0.8 \mathrm{~V}$; cone voltage, $3.0 \mathrm{~V}$; and sampling frequency, $2 \mathrm{~Hz}$. Ions were acquired in an MS acquisition range from m/z 100 to $\mathrm{m} / \mathrm{z} 650$ in continuum mode.

HPLC separations were achieved on two Chromolith Performance RP18e columns as first chromatographic system (100-4.6 mm; Merck KGaA) and Purospher Star RP18e Hibar HR column as second system (100-2.1 mm, $2 \mu \mathrm{m}$; Merck $\mathrm{KGaA}$ ) at a column oven temperature of $25^{\circ} \mathrm{C}$. For the first purification, elution was performed at a flow rate of $1.0 \mathrm{ml} / \mathrm{min}$ over a period of 30 minutes with a mixture of solvent $\mathrm{A}$ (water $+0.1 \%$ formic acid) and solvent $\mathrm{B}$ (acetonitrile $+0.1 \%$ formic acid). Compounds were eluted using the following conditions: mobile phase B increased from $0 \%$ to $25 \%$ over 7 minutes, followed by a 13 minute linear gradient to $60 \% \mathrm{~B}$ and a linear gradient to $100 \% \mathrm{~B}$ in 5 minutes, returning to $0 \% \mathrm{~B}$ in 0.1 minute and re-equilibration at $0 \% \mathrm{~B}$ for 4.9 minutes. The first 6 minutes of eluent were directed to waste to reduce contamination, as it contains mainly microsomes and buffer salts from incubation matrix. For the second purification, elution was performed at a flow rate of $0.45 \mathrm{ml} / \mathrm{min}$ over a period of 15 minutes, using the following conditions: mobile phase $B$ increased from $0 \%$ to $80 \%$ over 10 minutes, followed by linear gradient to $100 \% \mathrm{~B}$ in 2 minutes, returning to $0 \% \mathrm{~B}$ in 0.1 minute and re-equilibration at $0 \% \mathrm{~B}$ for 2.9 minutes. The eluate was subsequently split between the photodiode array detector and fraction manager $(98 \%)$ and the mass spectrometer $(2 \%)$. The MS flow was increased to $0.3 \mathrm{ml} / \mathrm{min}$ with a makeup solvent $(90 \%$ water $+10 \%$ acetonitrile). The fractionation of the analytes was triggered by the mass values $(\mathrm{m} / \mathrm{z} 265, \mathrm{~m} / \mathrm{z}$ 279 , and $\mathrm{m} / \mathrm{z} 425$ ) registered by the MS detector. Data acquisition and sample fractionation were performed using the software MassLynx 4.2 combined with FractionLynx.

Collected fractions were pooled, evaporated to dryness under nitrogen flow at $40^{\circ} \mathrm{C}$, and resolubilized in acetonitrile/water $(40 / 60 \%)$ for further purification. The fractions from the second purification step were evaporated to dryness for the soaking process.

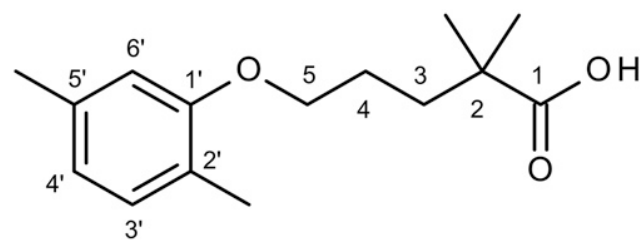

Fig. 1. Chemical structure of gemfibrozil. 
Ultra-Performance LC-MS ${ }^{2}$. The supernatants were analyzed on an Acquity UPLC Class I system (Waters Corporation) combined with a linear ion trap quadrupole (Qtrap 5500) mass spectrometer (AB Sciex LLC, Framingham). Samples were analyzed with an electrospray ion source in the negative ion mode. Source parameters were source temperature $600^{\circ} \mathrm{C}$ and ion spray voltage -4.5 $\mathrm{kV}$. Ultra-performance LC (UPLC) separations were achieved on an Acquity UPLC BEH C 18 column $(1.7 \mu \mathrm{m} ; 2.1 \times 50 \mathrm{~mm}$; Waters Corporation $)$ at a column oven temperature of $40^{\circ} \mathrm{C}$. Elution was performed at a flow rate of $0.7 \mathrm{ml} / \mathrm{min}$ over a period of 5.2 minutes with a mixture of solvent $\mathrm{A}$ (water $+0.1 \%$ formic acid + $10 \mathrm{mM}$ ammonium formate) and solvent B (acetonitrile). Compounds were eluted using the following conditions: mobile phase B increased from $30 \%$ to $50 \%$ between 0.1 and 4.0 minutes, followed by a 0.6-minute linear gradient to $100 \% \mathrm{~B}$, returning to $30 \% \mathrm{~B}$ in 0.02 minutes and re-equilibration at $30 \% \mathrm{~B}$ for 0.58 minutes. After injection, the column effluent was directed to the waste for 0.2 minutes to reduce contamination. The software Analyst 1.6.3 was used for data acquisition.

Crystalline Sponge Method. The porous crystalline sponge $\left[\left(\mathrm{ZnCl}_{2}\right)_{3}(\mathrm{tpt})_{2} \bullet \text { (cyclohexane) } \mathrm{x}\right]_{\mathrm{n}}$ was prepared according to the reported procedure (Biradha and Fujita, 2002; Ramadhar et al., 2015).

For guest soaking, one of the prepared $\left[\left(\mathrm{ZnCl}_{2}\right)_{3}(\mathrm{tpt})_{2} \bullet \mathrm{x} \text { (cyclohexane) }\right]_{\mathrm{n}}$ single crystals was used as crystalline sponge and then transferred with $50 \mu \mathrm{l}$ cyclohexane to the sample vial. Reference material was prepared as $1 \mathrm{mg} / \mathrm{ml}$ solution in DME, $1 \mu l$ of the solution pipetted into the sample vial, and the vial closed with a screw cap with septum seal (VWR, Darmstadt, Germany) and pierced with a syringe needle for slow evaporation of the solvent during guest inclusion. The samples were placed in an incubator at $50^{\circ} \mathrm{C}$ for 1 day. Soaking conditions for gemfibrozil glucuronide were conducted similarly. The volume of cyclohexane was reduced to $20 \mu \mathrm{l}$, and the incubator temperature was $25^{\circ} \mathrm{C}$. Incubation samples were prepared similarly to the reference material. Differences were that $40 \mu \mathrm{l}$ cyclohexane was transferred together with the crystal into the glass vial, which contained the pooled metabolite material. Four microliters of DME was added subsequently. The guest soaking for gemfibrozil glucuronide was performed using $20 \mu \mathrm{l}$ of cyclohexane at $25^{\circ} \mathrm{C}$ for 1 day.

Single crystal X-ray diffraction measurements were conducted by using a Rigaku Oxford Diffraction XtaLAB Synergy-R diffractometer, using $\mathrm{Cu}-\mathrm{K}_{\alpha}$ $\mathrm{X}$-ray radiation $\left(\lambda=1.54184 \AA\right.$ ) equipped with a HyPix-6000HE/HyPix-Arc $150^{\circ}$ Hybrid Photon Counting detector (Rigaku, Tokyo, Japan) at a temperature of $100 \mathrm{~K}$ with a Cryostream 800 nitrogen stream (Oxford Cryostreams, UK). The software CrysAlisPro (Rigaku Oxford Diffraction, 2018) was used for calculation of measurement strategy and data reduction (data integration, empirical and numerical absorption corrections, and scaling). Crystal structures were modeled using OLEX2 (Dolomanov et al., 2009), solved with SHELXT and refined by using SHELXL (Sheldrick, 2015). First, the refinement of the framework was performed by assigning atoms, modeling of disorder, applying anisotropic refinement, and adding of $\mathrm{H}$ atoms. The electron density of the guest molecules could safely be identified in the residual electron density after refinement of the framework. The amount of analyte in the sponge pores is modeled as occupancy of the respective atom coordinates, which is proportional to the observed electron density. The data quality of the measurement was assessed by $\mathrm{R}_{\mathrm{int}}$ and the refined model of framework and analyte by $R_{1}$. The $R_{1}$ value represents the agreement between calculated and observed model, and the $\mathrm{R}_{\mathrm{int}}$ is the measure of precision/reproducibility, classifying the recorded reflections from different angles.

The complete process from analyte generation to processing crystallographic data are shown in Fig. 2.

\section{Results}

LC-MS Analysis of Gemfibrozil Metabolites Prepared by Incubation with Rat/Human Liver Microsomes and Rat/Human Liver S9. Gemfibrozil (Fig. 1) was chosen as a tool compound to assess the ability of the CS to elucidate the complete structures of metabolites obtained from in vitro incubation. Hydroxylation reactions were conducted by hydroxylases present in microsomes, and further oxidation to carboxylic acid was performed by alcohol and aldehyde dehydrogenases present in the cytosol part of S9 fraction. Formation of gemfibrozil glucuronide was conducted by uridine diphosphoglucuronosyltransferase present in microsomes through conjugation with glucuronic acid (Zhang and Surapaneni, 2012) (Fig. 3).

After incubating gemfibrozil, the sample solution was tested with UPLC-MS-time-of-flight for metabolite identification. The settings applied on the MS-time-of-flight, did not allow for structure identification of neither the parent nor the metabolite. Further optimization was skipped, and the samples were rather analyzed on single quadrupole HPLC-MS and UPLC-MS ${ }^{2}$ in multiple-reaction monitoring mode.

The HPLC-MS chromatogram of gemfibrozil, incubated with rat liver microsomes and liver S9 fraction in the presence of NADPH or $\mathrm{NADPH} / \mathrm{NADP}^{+}$, showed the formation of two hydroxy- (M1 and M2) and one carboxy-gemfibrozil metabolite (M3). The three metabolites could not be observed in the absence of the cofactors, indicating that the metabolites were formed by enzymatic oxidation. All three metabolites detected in rat system were present in human liver S9 (Fig. 4). Gemfibrozil incubated with human liver microsomes in the presence of UDPGA showed the formation of a glucuronide (M4), which was only observable by addition of the cofactor. The metabolites M1 and M2 were detected at a deprotonated molecular mass $[\mathrm{M}-\mathrm{H}]^{-}$of $\mathrm{m} / \mathrm{z}$ 265. The $\mathrm{m} / \mathrm{z}$ value was $16 \mathrm{Da}$ higher than the $[\mathrm{M}-\mathrm{H}]^{-}$ion of gemfibrozil ( $\mathrm{m} / \mathrm{z} 249)$, suggesting the addition of one oxygen to the molecular structure. M3 was detected as deprotonated molecule $[\mathrm{M}-\mathrm{H}]^{-}$ at $\mathrm{m} / \mathrm{z} 279$, suggesting a further addition of oxygen and loss of two hydrogens but giving no information about the site of metabolism. M4 was detected at a deprotonated molecular mass $[\mathrm{M}-\mathrm{H}]^{-}$of $\mathrm{m} / \mathrm{z} 425$, indicating the addition of a glucuronic acid (176 Da).

To obtain more information, gemfibrozil and its metabolites were analyzed with UPLC-MS ${ }^{2}$. Tuning of instrument parameters provided the optimal transition for $4^{\prime}-\mathrm{OH}$ gemfibrozil reference material and was also applied for detection of M1 gemfibrozil. Precursor ion and product ion were adjusted to measure M3 gemfibrozil. Collision-induced dissociation of gemfibrozil and its glucuronide generated a main product ion of $\mathrm{m} / \mathrm{z} 121.0\left(2^{\prime}, 5^{\prime}\right.$-dimethylphenoxy moiety). As a result of the loss of the glucuronide moiety, both compounds were measured with identical transition $(\mathrm{m} / \mathrm{z} 249.1 / 121.0)$ but could be differentiated by their retention time. Hydroxygemfibrozil and carboxygemfibrozil were detected with $\mathrm{m} / \mathrm{z}$ values of 137.2 and 151.0, respectively (Fig. 4), indicating that oxidation $(+16 \mathrm{Da})$ and carboxylation $(+30 \mathrm{Da})$ occurred at the $2^{\prime}, 5^{\prime}$-dimethylphenoxy moiety and not at the chain of the carboxyalkyl group. This knowledge allowed to limit the metabolic position but could not give a more precise answer. However, CS-XRD provided precise information about the oxidation and glucuronidation sites as well as the structure of the metabolites.

Structural Elucidation of M1, M2, M3, and M4 Gemfibrozil by CS-XRD. A successful structure determination using the crystalline sponge method depends on various parameters, such as temperature, analyte concentration, duration of the soaking experiments, and solvent combinations during the soaking step (Hoshino et al., 2016). These parameters need to be optimized for each analyte. Different combinations of these parameters should be tried during this soaking process. Therefore, first analysis was conducted with $1000 \mathrm{ng}$ for each experiment of commercially available gemfibrozil, 4 '-hydroxy gemfibrozil, and gemfibrozil 1-O- $\beta$-glucuronide reference material to find the optimal soaking conditions for our tool compounds. The optimized soaking conditions were then applied to its metabolites generated from incubation. The reference material of $4^{\prime}$-hydroxy gemfibrozil was initially used to examine if this technology can be used for polar hydroxy and glucuronide metabolites.

Both the parent API and the reference metabolites 4 '-hydroxy gemfibrozil (Fig. 5B) and gemfibrozil 1-O- $\beta$-glucuronide could successfully be structurally elucidated by CS-XRD using an in-house XRD system. The crystal structure of gemfibrozil revealed two molecules with 


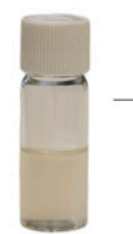

Day 1 In vitro incubation
Day 2-3 Sample preparation

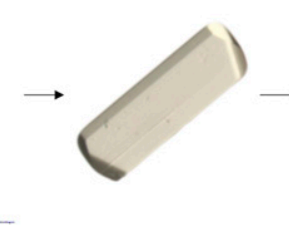

Day 4

Selection of crystalline sponge and sponge soaking

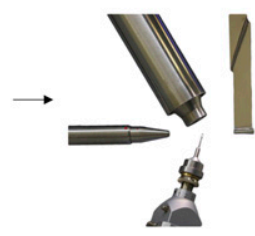

\section{Day 5}

Crystallography: Complete

structure elucidation

Fig. 2. Workflow of the metabolite identification processes using crystalline sponge technology.

occupancies of $100 \%$, and the final $\mathrm{R}_{1}$ and $\mathrm{R}_{\text {int }}$ values were $11.25 \%$ and $1.81 \%$, respectively. The crystal structure of gemfibrozil 1-O$\beta$-glucuronide revealed one molecule with occupancy of $100 \%$ and final $R_{1}$ and $R_{\text {int }}$ values of $6.99 \%$ and $1.87 \%$, respectively. Because of disorder, conformation of the glucuronide, i.e., the oxane ring and its substituents, cannot be determined. The presence of aliphatic rings in unfavorable conformations is often observed in the CS-XRD, especially for cyclohexane molecules. Nevertheless, the position of glucuronidation at the carboxylic acid function of gemfibrozil can be confirmed.

The successful results demonstrate that the application of the CS method can be expanded to the compounds containing highly polar hydrophilic groups introduced through metabolism of APIs, e.g., by using cytochrome P450 enzymes, despite the hydrophobic pore of the CS.

Next, the CS method was applied to metabolites obtained by incubation. Incubating gemfibrozil with rat liver microsomes for M1 and M2, rat liver S9 for M3, and human liver microsomes for M4 resulted in the formation of four metabolites in different quantities as described above. The reference material of $4^{\prime}-\mathrm{OH}$ gemfibrozil and gemfibrozil 1-O- $\beta$-glucuronide allowed the quantification of M2 and
M4, whereas the amounts of M1 and M3 were estimated. For pooling of M1, M3, and M4, an incubation solution of $20 \mu \mathrm{M}$ gemfibrozil was used, and $200 \mu \mathrm{M}$ gemfibrozil was used for M2. The direct use of the separated metabolites was not possible because of the sensitivity of the CS-XRD against impurities as already mentioned in previous publications (Inokuma et al., 2016; Kai et al., 2018). The impurities resulting from the incubation matrix that is necessary for preparation of metabolites by enzymatic reaction (e.g., salts, cofactor, microsomal stock solution) could not be separated by using only one purification step. Therefore, the pooled samples were further purified using a second column. CS soaking experiments were conducted with final amounts in a range of 500-1000 ng per experiment.

The soaking experiments of the obtained metabolites M1-M4 were carried out under the same conditions as used for the reference material. The soaked CS were then measured via XRD, and the crystallographic analysis clearly revealed the positions of hydroxylation, carboxylation, and glucuronidation.. M1 exhibits relatively high electron density in close proximity to the 5 '-methyl carbon atom forming a benzyl alcohol, which is further oxidized at the same position to form a benzoic acid (M3). M2 shows high electron density at position $4^{\prime}$ of the $2^{\prime}, 5^{\prime}$-dimethylphenoxy

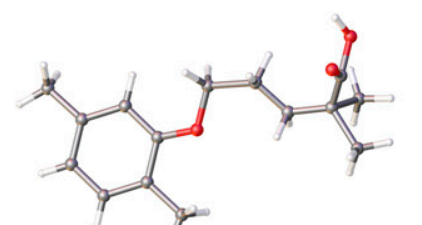<smiles>Cc1ccc(C)c(OCCCC(C)(C)C(=O)O)c1</smiles>

Gemfibrozil
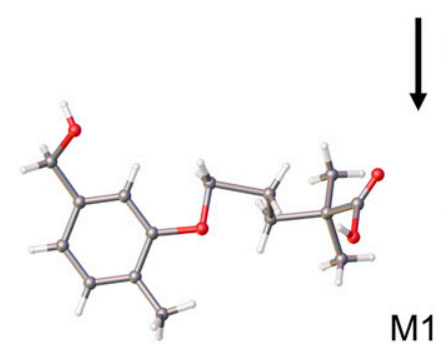
Microsomes/S9<smiles>Cc1ccc(CO)cc1OCCCC(C)(C)C(=O)O</smiles>
s9

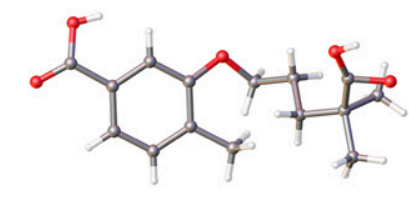<smiles>Cc1ccc(C(=O)O)cc1OCCCC(C)(C)C(=O)O</smiles><smiles>Cc1ccc(C)c(OCCCC(C)(C)C(=O)OC2OC(C(=O)O)C(O)C(O)C2O)c1</smiles>

M4

Fig. 3. Metabolic pathway of gemfibrozil; $\mathrm{M} 1,5^{\prime}-\mathrm{CH}_{2} \mathrm{OH}$ gemfibrozil; M2, 4'-OH gemfibrozil; M3, 5'-COOH gemfibrozil; M4, gemfibrozil glucuronide. 
A

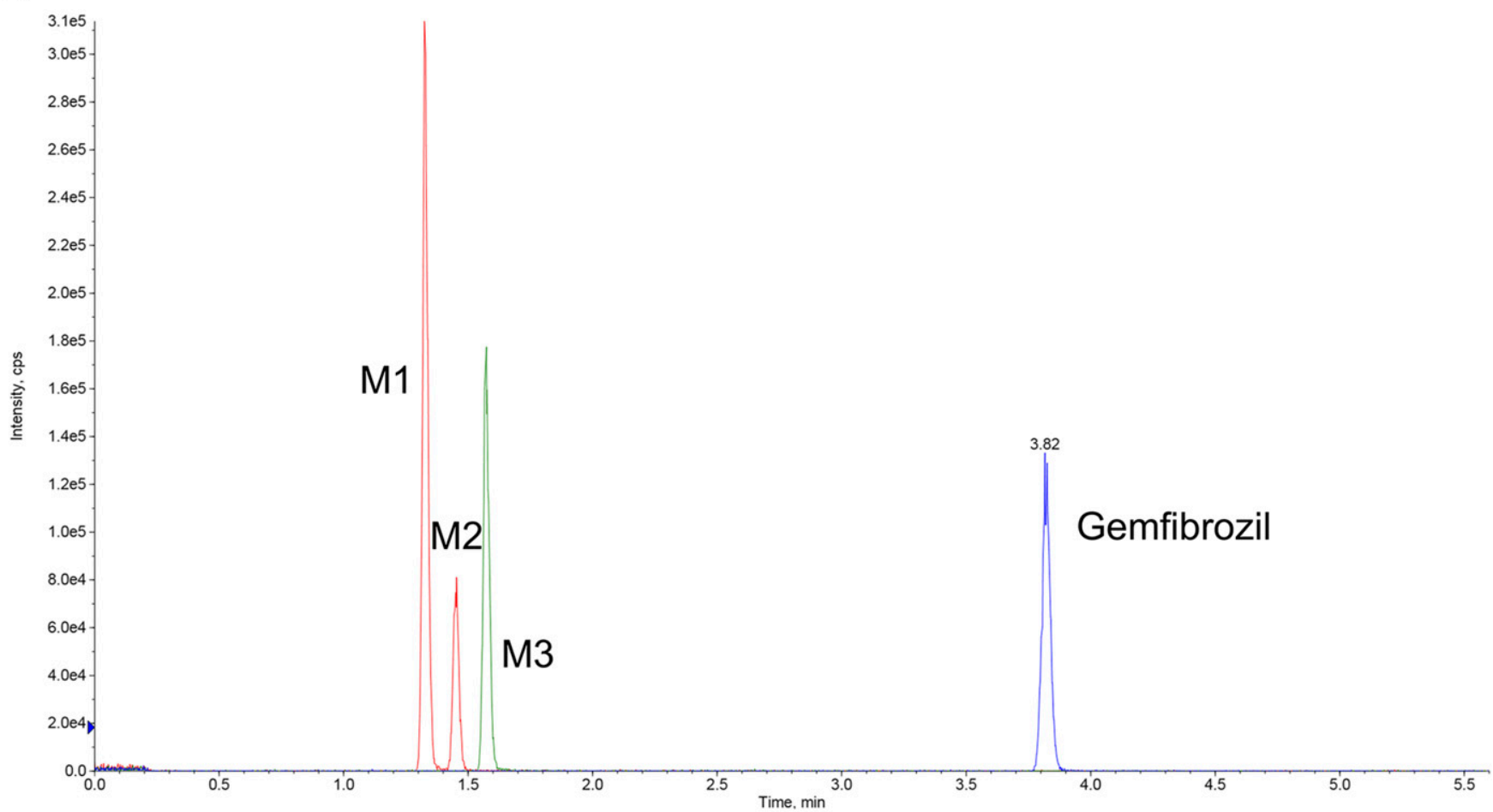

B

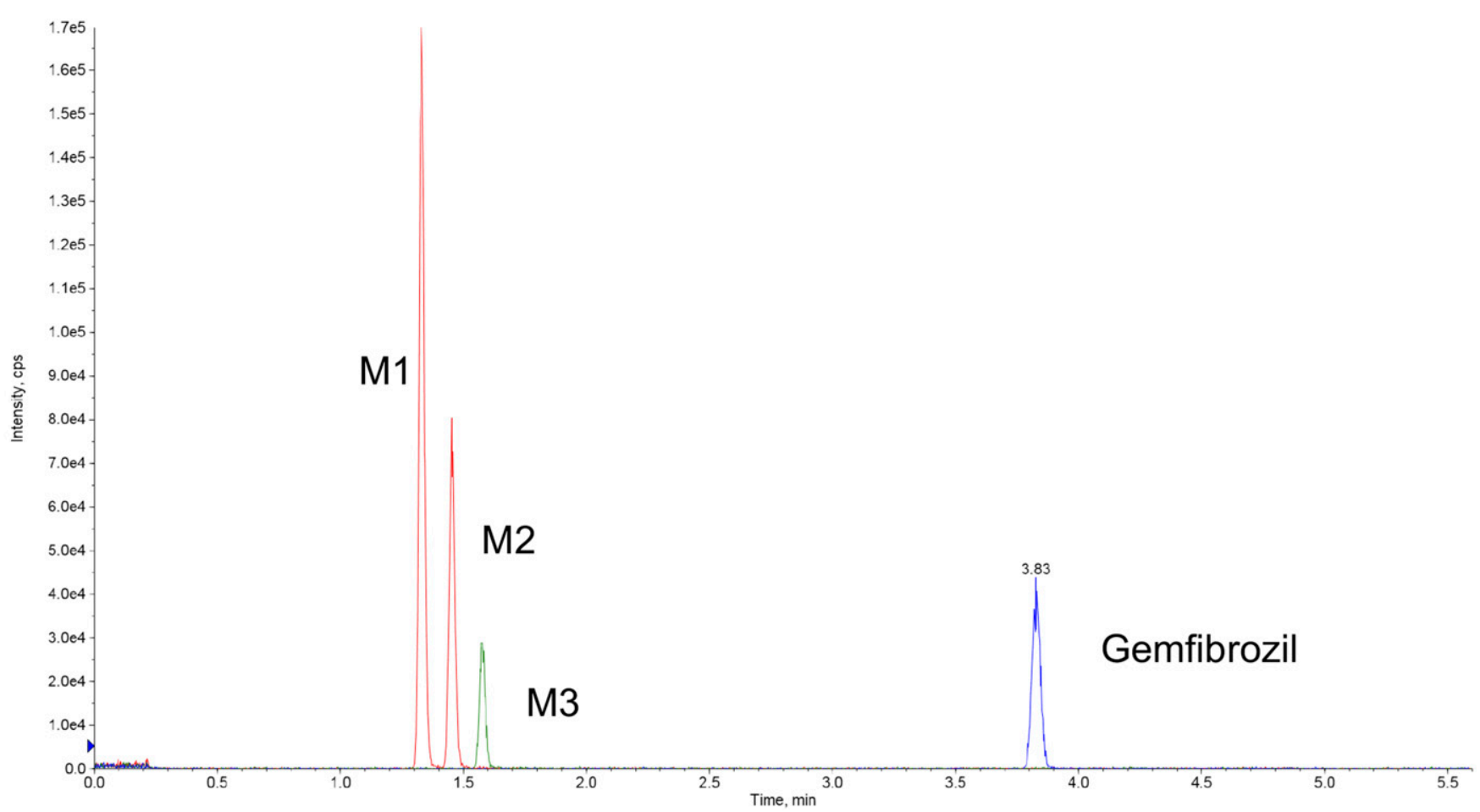

Fig. 4. Extracted ion chromatogram of multiple reaction monitoring (MRM) for gemfibrozil with rat liver S9 (A) and human liver S9 (B) in the presence of NADPH and $\mathrm{NADP}^{+}$as cofactors. M1 (5'- $\mathrm{CH}_{2} \mathrm{OH}$ gemfibrozil): $\mathrm{m} / \mathrm{z}$ 265.0/137.2; M2 (4'-OH gemfibrozil): $\mathrm{m} / \mathrm{z}$ 265.0/137.2; M3 (5'-COOH gemfibrozil): $\mathrm{m} / \mathrm{z}$ 279.0/151.0; gemfibrozil: $\mathrm{m} / \mathrm{z} 249.1 / 121.0$

moiety. The electron density map of the benzene core from gemfibrozil and its metabolites M1-M3 are shown in Fig. 5B. Two molecules of 5' hydroxymethyl gemfibrozil and one cyclohexane were clearly assigned by its electron density in the asymmetric unit with occupancies of
$100 \%$ (Fig. 5A). The modeled structure shows a $\mathrm{R}_{1}$ value of $7.58 \%$ and a value of $2.18 \%$ for $\mathrm{R}_{\text {int }}$. One guest is stabilized by $\pi$ - $\pi$ stacking interactions between its aromatic ring and the tpt ligand of the framework (distance $3.63 \AA$, angle $5.83^{\circ}$ ). Crystallographic data of $4^{\prime}$-hydroxy 
A

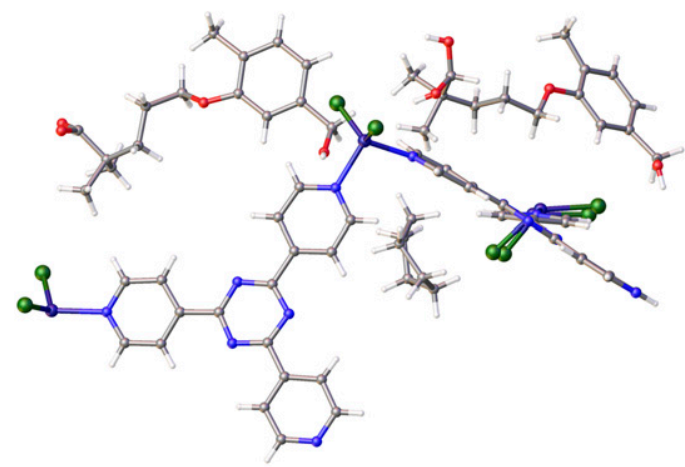

B

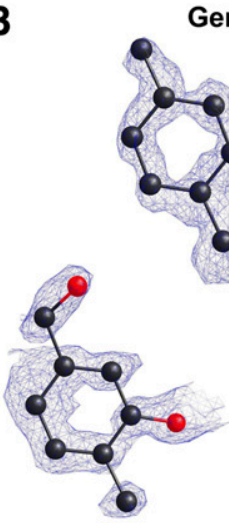

M1
Gemfibrozil

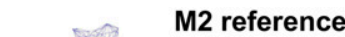

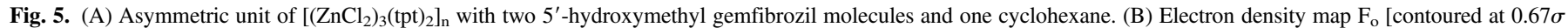
(gemfibrozil), 0.65 $(\mathrm{M} 2$ reference), $0.73 \sigma(\mathrm{M} 1), 0.95 \sigma(\mathrm{M} 2), 1.00 \sigma(\mathrm{M} 3)]$ of benzene core from reference material and incubation samples.

gemfibrozil revealed one analyte molecule with occupancy of $44 \%\left(\mathrm{R}_{1}\right.$ $7.56 \%, \mathrm{R}_{\text {int }} 2.09 \%$ ) and two cyclohexane molecules. M2 interacts with the framework by $\pi-\pi$ stacking interactions between the aromatic ring and the tpt ligand (distance $3.54 \AA$, angle $7.90^{\circ}$ ) as well as $\mathrm{CH} \cdots \mathrm{Cl}$ interactions (distance $2.50 \AA$ ) between the $5^{\prime}$-methylgroup of the analyte and the $\mathrm{ZnCl}_{2}$ part of the framework (Fig. 6). The data of $5^{\prime}$ carboxygemfibrozil provided low electron density of the analyte but clearly confirmed the position of metabolism in comparison with the crystal structure obtained from 5'-hydroxymethylgemfibrozil (Fig. 5B). The XRD data of M4 showed the formation of an acyl glucuronide by conjugation of the carboxylic acid moiety of gemfibrozil with the C1hydroxy group of glucuronic acid. This confirmed the position of glucuronidation, but because of low electron density of the analyte, the reference material was used to illustrate the structure in this publication. M3 and M4 could be assigned with low electron density compared with the hydroxy metabolites because of the higher polar and nucleophilic groups. The refined structures of gemfibrozil and its generated metabolites are shown in Fig. 3. Crystallographic data and ORTEP diagrams of the asymmetric unit of the framework and analyte are shown in the Supplemental Data.

\section{Discussion}

In summary, we were able to analyze the structure of three different phase I metabolites and one phase II acyl glucuronide of our tool

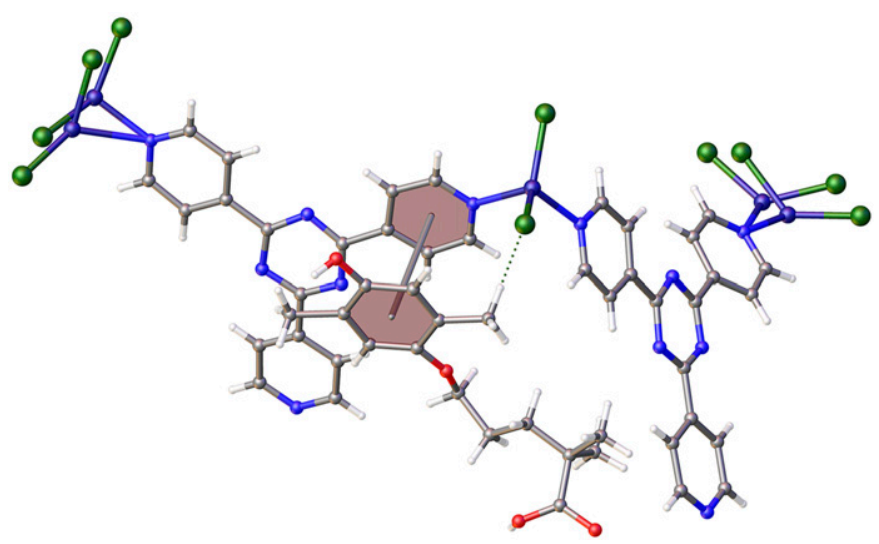

Fig. 6. The crystal structure of M2 gemfibrozil exhibits $\pi-\pi$ stacking interactions (gray line) and $\mathrm{CH} \cdots \mathrm{Cl}$ interactions (green dashed line) between the analyte and the framework. Centroids are shown as gray spheres in the center of the benzene ring. compound gemfibrozil at atomic level with only nanogram amounts of substance by using the crystalline sponge method. Data from MS analysis provided us information on the type of metabolism but could not pinpoint the exact position of the hydroxylation, carboxylation, or glucuronidation reaction. Only the crystallographic data showed that hydroxylation occurred at the $4^{\prime}-\mathrm{C}$ and $5^{\prime}-\mathrm{CH}_{3}$ positions of the aromatic ring as well as further oxidation at position $5^{\prime}-\mathrm{CH}_{3}$ to form a carboxylic acid and direct glucuronidation at the carboxylic acid moiety of the alkyl chain. The Cambridge Crystallographic Data Centre did not contain crystallographic data of an acyl glucuronide before and could be extended through M4 gemfibrozil.

The need to identify the structure of metabolites meets the need of industry to assess the contribution of drug metabolites to efficacy, toxicity, or drug-drug interactions. It also meets the requirements from regulatory agencies to ascertain that human metabolites are present in preclinical species used to assess drug safety. Acyl glucuronides especially play an important role in the assessment of drug-related risk factors, as they contain the risk to be potentially reactive metabolites. An intramolecular rearrangement process, so called "migration," leads to the formation of chemically reactive species that can covalently bind proteins (Bailey and Dickinson, 2003; Zhong et al., 2015).

The application of the crystalline sponge technology in structure elucidation is a convenient technology that can be applied in drug discovery to accelerate the metabolite identification processes and allow for using human-derived systems to identify human metabolites from in vitro incubation, years before clinical samples are available.

This technology is, however, in its infancy. Therefore, optimization of soaking conditions (time, solvent, and temperature) as well as assessing the purity required for compound is still needed. The amount of analyte per experiment was used in excess but could be reduced as in the original publication mentioned (Inokuma et al., 2013). It has also been described that not all compounds can be applied in this technology, as the size of the molecules may prevent them from entering the pores and their polarity may prevent some molecules to dissolve in the hydrophobic solvents typically used during soaking. It was therefore unclear if the technology would be applicable to drug metabolites resulting from phase I and especially phase II reactions. To answer this question, we used reference metabolites (M2 and M4 gemfibrozil) to confirm the ability of CS-XRD to assess the structure of phase I and phase II metabolites. Beside these two metabolites, we have applied this new technology to identify the structure of the two other unknown metabolites (M1 and M3 
gemfibrozil), confirming its application in drug development of new chemical entities for which only the structure of the candidate molecule is known.

Our results show that the combination of CS-XRD with MS data offers a great opportunity for scientists in drug metabolism and pharmacokinetics to assess the structure of metabolites produced in low amounts from in vitro studies. The technology is also able to provide information on stereochemistry, offering a significant advantage over other techniques such as MS or NMR. In conclusion, we show that CS can be used to assess the structure of traces of drug-like molecules as well as their metabolites, showing promising application at different stages of drug development when complete structure elucidation is needed. Beside metabolites, this technology could also be applied to identify degradation products and impurities during drug product development.

\section{Acknowledgments}

The authors thank Andrea Unzue-Lopez for kindly providing 4'-OH gemfibrozil reference material and Axel-Walter Thomasberger, Ralf-Erwin Licht, Jonny Nachtigall, and Birikiti Kidane for their kind technical support in the laboratory. The authors also thank Anna K.H. Hirsch for her constructive comments of the manuscript.

\section{Authorship Contributions}

Participated in research design: All authors.

Conducted experiments: Rosenberger, von Essen, Khutia.

Contributed new reagents or analytic tools: Kühn, Urbahns.

Performed data analysis: Rosenberger, von Essen.

Wrote or contributed to the writing of the manuscript: Rosenberger, von Essen, Khutia, Kühn, Hartmann, Badolo.

\section{References}

Bailey MJ and Dickinson RG (2003) Acyl glucuronide reactivity in perspective: biological consequences. Chem Biol Interact 145:117-137.

Biradha K and Fujita M (2002) A springlike 3D-coordination network that shrinks or swells in a crystal-to-crystal manner upon guest removal or readsorption. Angew Chem Int Ed Engl 41: 3392-3395.

Brandon EFA, Raap CD, Meijerman I, Beijnen JH, and Schellens JHM (2003) An update on in vitro test methods in human hepatic drug biotransformation research: pros and cons. Toxico Appl Pharmacol 189:233-246.

Dolomanov OV, Bourhis LJ, Gildea RJ, Howard JAK, and Puschmann H (2009) OLEX2: a complete structure solution, refinement and analysis program. J Appl Cryst 42:339-341.

EMA (2013) Guideline on the Investigation of Drug Interactions, European Medicines Agency, Amsterdam, Netherlands.
Fasinu P, Bouic PJ, and Rosenkranz B (2012) Liver-based in vitro technologies for drug biotransformation studies - a review. Curr Drug Metab 13:215-224.

FDA (2016) Guidance for Industry: Safety Testing of Drug Metabolites, US Department of Health and Human Services FDA, Center for Drug Evaluation and Research, Silver Spring, MD.

Hoshino M, Khutia A, Xing H, Inokuma Y, and Fujita M (2016) The crystalline sponge method updated. IUCrJ 3:139-151.

Inokuma Y, Ukegawa T, Hoshino M, and Fujita M (2016) Structure determination of microbial metabolites by the crystalline sponge method. Chem Sci (Camb) 7:3910-3913.

Inokuma Y, Yoshioka S, Ariyoshi J, Arai T, Hitora Y, Takada K, Matsunaga S, Rissanen K, and Fujita M (2013) X-ray analysis on the nanogram to microgram scale using porous complexes [published correction appears in Nature (2013) 495:461-466]. Nature 501:262.

Jones CG, Martynowycz MW, Hattne J, Fulton TJ, Stoltz BM, Rodriguez JA, Nelson HM, and Gonen T (2018) The CryoEM method MicroED as a powerful tool for small molecule structure determination. ACS Cent Sci 4:1587-1592.

Kai K, Sogame M, Sakurai F, Nasu N, and Fujita M (2018) Collimonins A-D, unstable polyynes with antifungal or pigmentation activities from the fungus-feeding bacterium collimonas fungivorans Ter331. Org Lett 20:3536-3540.

Kunde T and Schmidt BM (2019) Microcrystal electron diffraction (MicroED) for small-molecule structure determination. Angew Chem Int Ed Engl 58:666-668.

Ma S and Chowdhury SK (2011) Analytical strategies for assessment of human metabolites in preclinical safety testing. Anal Chem 83:5028-5036.

Massa W (2004) Crystal Structure Determination, Springer-Verlag GmbH, Heidelberg, Germany. Morita I, Mori T, Mitsuhashi T, Hoshino S, Taniguchi Y, Kikuchi T, Nagae K, Nasu N, Fujita M Ohwada T, et al (2020) Exploiting a C-N bond forming cytochrome P450 monooxygenase for C-S bond formation. Angew Chem Int Ed Engl 59:3988-3993.

Murai T, Iwabuchi H, and Ikeda T (2004) Identification of gemfibrozil metabolites, produced as positional isomers in human liver microsomes, by on-line analyses using liquid chromatography/ mass spectrometry and liquid chromatography/nuclear magnetic resonance spectroscopy. J Mass Spectrom Soc Jpn 52:277-283.

Nedderman AN (2009) Metabolites in safety testing: metabolite identification strategies in discovery and development. Biopharm Drug Dispos 30:153-162.

Prakash C, Shaffer CL, and Nedderman A (2007) Analytical strategies for identifying drug metabolites. Mass Spectrom Rev 26:340-369.

Prasad B, Garg A, Takwani H, and Singh S (2011) Metabolite identification by liquid chromatography-mass spectrometry. Trends Analyt Chem 30:360-387.

Ramadhar TR, Zheng SL, Chen YS, and Clardy J (2015) Analysis of rapidly synthesized guestfilled porous complexes with synchrotron radiation: practical guidelines for the crystalline sponge method. Acta Crystallogr A Found Adv 71:46-58.

Rigaku Oxford Diffraction (2018) CrysAlis PRO, Rigaku Corporation, Tokyo, Japan.

Sakurai F, Khutia A, Kikuchi T, and Fujita M (2017) X-ray structure analysis of n-containing nucleophilic compounds by the crystalline sponge method. Chemistry 23:15035-15040.

Sheldrick GM (2015) Crystal structure refinement with SHELXL. Acta Crystallogr C Struct Chem 71:3-8.

Wada N, Kersten RD, Iwai T, Lee S, Sakurai F, Kikuchi T, Fujita D, Fujita M, and Weng JK (2018) Crystalline-sponge-based structural analysis of crude natural product extracts. Angew Chem Int Ed Engl 57:3671-3675.

Zhang D and Surapaneni S (2012) ADME-Enabling Technologies in Drug Design and Development, John Wiley \& Sons, Inc., Hoboken, New Jersey.

Zhang Z, Zhu M, and Tang W (2009) Metabolite identification and profiling in drug design: current practice and future directions. Curr Pharm Des 15:2220-2235.

Zhong S, Jones R, Lu W, Schadt S, and Ottaviani G (2015) A new rapid in vitro assay for assessing reactivity of acyl glucuronides. Drug Metab Dispos 43:1711-1717.

Address correspondence to: Dr. Lassina Badolo, Merck KGaA, Frankfurter Str. 250, 64293 Darmstadt, Germany. E-mail: lassina.badolo@emdserono.com 